\title{
Comparative analysis of the efficiency of gene expression of bacterial D-lactate dehydrogenases in yeast Schizosaccharomyces pombe
}

\author{
Anisimova E.O. ${ }^{1,2 *}$, Blokhina K.S. ${ }^{2}$, Tarutina M.G. ${ }^{1,2}$, Sineoky S.P. ${ }^{2}$ \\ ${ }^{1}$ NRC «Kurchatov Institute»- GOSNIIGENETIKA, Kurchatov Genomic Center, NRC «Kurchatov Institute», Moscow, Russia \\ ${ }^{2} N R C$ «Kurchatov Institute», Moscow, Russia \\ *e-mail: ekaterina.genetika@gmail.com
}

Key words: Schizosaccharomyces pombe, D-lactic acid, gene expression

Motivation and Aim: Lactic acid (LA) is a platform chemical for the sustainable production of various materials. In recent years, there has been a growing interest in poly lactic acid (PLA) as a biodegradable polymer that is chemically synthesized from LA produced by microbiological process from renewable resources. There are two enantiomers of lactic acid: L-LA and D-LA. Both optical isomers are required to produce PLA with improved physical properties (thermochemical properties and higher rate of PLA biodegradation), which can be achieved by creating a stereo complex PLA with different combination ratios of the optically pure L-LA and D-LA isomers [1]. The production of optically pure D-LA has commercial attention because of the rapid growth of the PLA industry.

Methods and Algorithms: An acid-tolerant to high concentrations of lactic acid the S. pombe strain was used as recipient for a low-pH $D$-LA production. The $S$. pombe strain doesn't produce $D$-LA, so there were constructed the genetically modified $S$. pombe strains containing $D-L D H$ genes from Leuconostoc mesenteroides [2], Lactobacillus delbrueckii [3] и Lactobacillus plantarum [4] - bacterial producers of highly stereospecific $D$-LA. Recombinant strains were cultivated in tubes and the concentrations of $D$-LA were measured.

Results: The $S$. pombe strain expressing $D-L D H$ gene from $L$. plantarum produced more $D$-LA in comparison with strains expressing two other $D-L D H$ genes.

Conclusion: This investigation can be used to construct a highly effective strain that produces $D$-LA under acidic fermentation conditions.

Acknowledgements: Supported by the Ministry of Science and Higher Education of the Russian Federation (Grant No. 075-15-2019-1658), and was performed using the resources of the Bioresource Center - AllRussian Collection of Industrial Microorganisms.

\section{References}

1. Wang, Y., Tashiro, Y. and Sonomoto, K. Fermentative production of lactic acid from renewable materials: Recent achievements, prospects, and limits. Journal Bioscience and Bioengineering. 2015;119(1):10-18.

2. Seung-Ho Baek et al. Improvement of D-Lactic acid production in Saccharomyces cerevisiae under acidic conditions by evolutionary and rational metabolic engineering, Biotechnol Journal. 2017;12(10).

3. Caili Zhang et al. Non-sterilized fermentation of highoptically pure d-lactic acid by a genetically modified thermophilic Bacillus coagulans strain, Microbial Cell Factories. 2017;16(1):213.

4. Kenji Okano et al. Efficient production of optically pure D-lactic acid from raw corn starch using genetically modified L-lactate dehydrogenase gene-deficient and $\alpha$-amylase secreting, Applied Environmental Microbiol. 2009;75(2):462467. 